\title{
Presurgical MRSA Screening and Subsequent Decolonization in Elective Instrumented Neurosurgery: A Case Descriptive Study
}

\author{
Vincent De La Porte ${ }^{1}$, Veroniek Saegeman ${ }^{2}$, Elise Willems ${ }^{2}$, Charlotte Stolte ${ }^{3}$ and Erik Van de Kelft ${ }^{4,5 *}$ \\ ${ }^{1}$ University Hospital Antwerp, Wilrijkstraat 10, 2650 Edegem, Belgium \\ ${ }^{2}$ Department of Microbiology, AZ Nikolaas, Moerlandstraat 1, 9100, Sint-Niklaas, Belgium \\ ${ }^{3}$ Department of Anesthesiology, AZ Nikolaas, Moerlandstraat 1, 9100, Sint-Niklaas, Belgium \\ ${ }^{4}$ Department of Neurosurgery, AZ Nikolaas, Moerlandstraat 1, 9100, Sint-Niklaas \\ ${ }^{5}$ Antwerp University, Faculty of Medicine and Health Sciences, Wilrijk, Belgium \\ ${ }^{*}$ Corresponding author: Prof. dr. Erik Van de Kelft, Department of Neurosurgery, AZ Nikolaas, Moerlandstraat 1, 9100, Sint-Niklaas, Belgium; Tel: + 3237602172 ; \\ E-mail: Erik.VandeKelft@aznikolaas.be
}

Received: October 23, 2019; Accepted: December 01, 2019; Published: January 07, 2020;

\begin{abstract}
Background

Staphylococcus aureus is one of the main microorganisms causing a surgical site infection. Methicillin resistant staphylococcus aureus (MRSA) surgical site infection treatment may be difficult, requires long-term antibiotic treatment, especially in cases of instrumented procedures, deteriorates the clinical result, and generates a high medical and social cost. Preoperative colonization with MRSA is a risk factor of SSI's
\end{abstract}

\section{Purpose}

To identify the prevalence of MRSA colonization of patients scheduled for elective and instrumented neurosurgery and the success rate of current MRSA decolonization protocol. Many studies have addressed MRSA colonization rate and impact of MRSA decolonization. However, large studies on this topic in elective and instrumented neurosurgery are sparse.

\section{Materials and methods}

A total of 1749 patients, scheduled for elective instrumented neurosurgery, were included for this study. All patients were screened for MRSA and those who were diagnosed as MRSA carriers underwent decontamination preoperatively. The medical files of the patients, who screened MRSA positive, were searched for risk factors of MRSA carriage. In addition, information of postsurgical MRSA positive cultures was collected for detection of surgical site infection caused by MRSA.

\section{Results}

The colonization rate of patients scheduled for elective instrumented neurosurgery was $0.74 \%$. After completing the first decontamination protocol, all MRSA carriage was eliminated. At least one risk factor for MRSA carriage was reported for 11 of the 13 patients, MRSA carriers. One patient, who was preoperatively MRSA negative, had an MRSA surgical site infection.

\section{Conclusion}

The prevalence of MRSA carriage in elective, instrumented neurosurgery was $0.74 \%$ and no resistance was observed against our decolonization protocol. Preoperative MRSA screening can be used to reduce possible complications caused by MRSA after surgery. The preferred strategy for screening and decontamination depends on several, mostly local factors.

Keywords: MRSA screening; MRSA prevalence; MRSA decolonization; Surgical Site Infection; neurosurgery; screening strategy

\section{Introduction}

Staphylococcus aureus is one of the main microorganisms causing a surgical site infection (SSI). Methicillin resistant staphylococcus aureus (MRSA) SSI treatment may be difficult, requires long-term antibiotic treatment, especially in cases of instrumented procedures, deteriorates the clinical result, and generates a high medical and social cost [1]. Preoperative colonization with MRSA is a risk factor of SSI's [2]. 
S. aureus and MRSA are most frequently identified in the anterior nares. Other common extra-nasal sites are the skin, pharynx and perineum [3]. Several risk factors for MRSA carriage have been identified in the literature and are listed in Table 1 [4-13].

Table 1. Risk factors for MRSA carriage

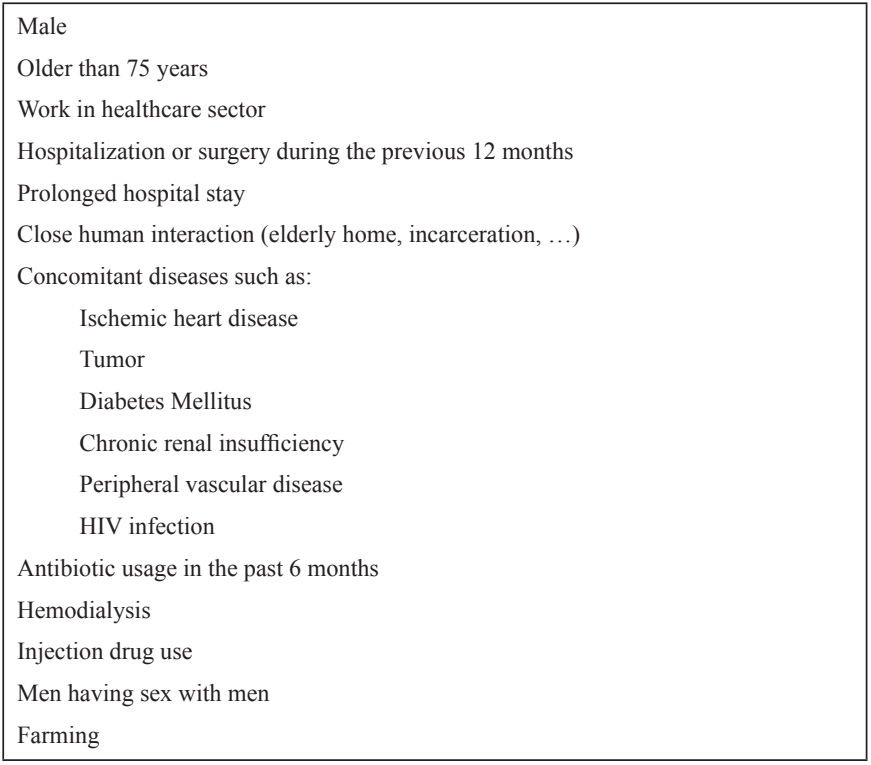

*MRSA: methicillin resistant staphylococcus aureus; HIV: human immunodeficiency virus

Preoperative screening and subsequent decolonization of MRSA positive patients prior to surgery is a well-known strategy to reduce the risk of SSI's with MRSA [14].

Although systematic screening and targeted decolonization involves an additional cost and effort, it may reduce expenditures due to a decrease in complications correlated with MRSA infection [15].

This study examines (a) the prevalence of MRSA colonization in a population of patients scheduled for elective and instrumented neurosurgery, (b) the success rate of MRSA decolonization, (c) the occurrence of a SSI in patients who underwent elective surgery and (d) the presence of risk factors (Table 1) for MRSA carriage in the colonized group.

\section{Materials and Methods}

From April 2012 until June 2015, preoperative screening for MRSA carriage was performed in 1997 patients who were scheduled for a neurosurgical procedure in AZ Nikolaas. All urgent and noninstrumented interventions were excluded for this study, which resulted in a final database including 1749 elective and instrumented neurosurgical procedures. Most frequent procedures were posterior lumbar interbody arthrodeses (772), anterior cervical discectomies and arthrodeses (531), screw implantations (129) and anterior lumbar interbody arthrodeses (125). MRSA screening was performed 2 to 3 weeks prior to surgery either at the clinical laboratory of AZ Nikolaas, the general practitioner's office, at home or abroad. At the clinical laboratory of AZ Nikolaas, screening was performed at three different body sites (anterior nares, pharynx and perineum). These samples were obtained by using swabs suitable for aerobes and anaerobes (Nuova Aptaca, Canelli, Italy). Screening that was performed at AZ Nikolaas was at a cost of $€ 21.6$ per patient. Screening outside the hospital (at general practitioner's office, at home or screening abroad) involved a swab of the anterior nares and was performed at an unknown cost.

Within 24 hours of sampling, swabs were soaked in enrichment broth containing $\mathrm{NaCl}$ 6,5\% and streaked onto ChromID MRSA (bioMérieux, Marcy l' Etoile, France). These agar plates contain cefoxitin to inhibit oxacillin susceptible $S$. aureus strains. The agar plate was incubated for $24-48 \mathrm{hrs}$ at $35 \pm 2^{\circ} \mathrm{C}$ in ambient air. Growth of green colonies was suspected for MRSA, these colonies were identified by means of mass spectrometry (Bruker Biotyper, Germany). S. aureus strains were confirmed for their resistance to cefoxitin by disk diffusion (ROSCO tablets) using EUCAST antimicrobial breakpoint criteria.

In case of a positive MRSA screening, the surgeon contacted the patient to ask his approval for an MRSA decolonization procedure. This decolonization procedure implies: a $2 \%$ mupirocin ointment which was topically applied to the anterior nares 3 times daily for 5 days, daily bathe and 1-2 hair washes with $40 \mathrm{mg} / \mathrm{ml}$ chlorhexidine glucoronate solution or $10 \%$ povidone-iodine gel for 5 days. On the $8^{\text {th }}$ day after the start of the decolonization protocol, a new MRSA screening was executed. If tested negative, two consecutive screenings took place on day 10 and 11. If tested positive, a second decolonization was performed. Surgery was performed when the patient initially screened negative or in case of three consecutive negative screening results after the MRSA decolonization procedure. The total cost of a decolonization procedure with three consecutive screenings was $€ 89.3$ per patient.

The medical records of presurgical MRSA positive patients were searched for the presence of risk factors shown in Table 1. We registered the results of all MRSA screenings, if screening was carried out in the hospital and the occurrence of SSI caused by MRSA. Furthermore, data of all pre- and postsurgical positive MRSA cultures of neurosurgical patients were collected, for the same time period, from the database of the department of Microbiology. All data were compiled using Excel (Version 15.19.1, 2016, Microsoft, Redmond, WA, USA) for subsequent analyses. This study was approved by the medical ethics committee of AZ Nikolaas, reference number EC17023.

\section{Results}

The MRSA colonization rate in our patients undergoing elective instrumented neurosurgery was $0.74 \%$ (13 of 1749). Of all screenings, 1227 (70.2\%) were performed in AZ Nikolaas and 522 (29.8\%) outside the hospital. Of the thirteen MRSA positive screenings, eight (62\%) were performed in the hospital lab and five (38\%) in other labs. The detection rate using samples from three different body sites was $0.65 \%$ ( 8 of 1227 ) and $0.96 \%$ (5 of 522) in case only the anterior nares were sampled.

After completing the first decolonization protocol, none of the initially MRSA positive patients screened positive again.

Of the thirteen pre-surgical MRSA positive patients, twelve medical files included information about risk factors for MRSA 
carriage. This search revealed that three patients were male, one was older than 75 years, two were active in the healthcare sector at the moment of surgery and three were working on a farm. In the period of 12 months prior to surgery, three patients had been hospitalized and two of them had undergone surgery.

Assessing the underlying diseases, one patient was known with diabetes mellitus, one with chronic kidney disease and two with peripheral vascular disease (Table 2 ).

Table 2. Presence of risk factors in MRSA positive patients

\begin{tabular}{|l|c|c|}
\hline \multicolumn{1}{|c|}{ Risk factor* } & Number of patients & $\%$ \\
\hline Male & 3 & $25 \%$ \\
\hline Older than 75 years & 1 & $8 \%$ \\
\hline Active in healthcare sector & 2 & $17 \%$ \\
\hline Farming & 3 & $25 \%$ \\
\hline Hospitalization previous 12 months & 3 & $25 \%$ \\
\hline Surgery previous 12 months & 2 & $17 \%$ \\
\hline Diabetes mellitus & 1 & $8 \%$ \\
\hline Chronic kidney disease & 1 & $8 \%$ \\
\hline Peripheral vascular disease & 2 & $17 \%$ \\
\hline
\end{tabular}

* One patient can have more than one risk factor; MRSA: methicillin resistant staphylococcus aureus

At least one risk factor was present in eleven (92\%) MRSA colonized patients. There were no declared relations with the other assessed risk factors.

No surgical site infection occurred in the preoperative MRSA carrying and decolonized patients. Of all patients who underwent instrumented neurosurgery, one preoperative MRSA negative patient had a deep incisional MRSA SSI, 48 hours after operation, for which reoperation was necessary.

\section{Discussion}

To determine the role of MRSA screening in elective instrumented neurosurgery, defining the prevalence of MRSA colonization and MRSA related SSI is essential. Studies identifying preoperative MRSA colonization in elective instrumented neurosurgery are scarce and the local MRSA prevalence must be taken into consideration when interpreting the results.

This study included data of 1749 patients and found a MRSA colonization rate of $0.74 \%$. This is comparable to the reported MRSA prevalence of $0.4 \%$ in Belgium [16].

None of the 13 preoperative MRSA positive patients had an SSI. However, one preoperative MRSA negative patient had an SSI caused by MRSA. Although the carriage of MRSA is a known risk factor for surgical site infections with MRSA, Kawabata et al. reported no added value of MRSA nasal swab cultures in spinal surgery for predicting SSI [17].

Evaluation of the screening was only performed for MRSA and not for methicillin sensitive Staphylococcus aureus (MSSA). A significant part of surgical site infections is caused by MSSA and screening for MSSA followed by decolonization may result in a greater reduction of SSI [18].

An important aspect in MRSA screening is the anatomical site sampled for screening. Screening from multiple body sites such as a combination of nasal, throat and perineal or groin sampling perform better than nasal screening alone [19]. We could not verify this statement in our study since the MRSA colonization rate was slightly lower when screening was performed at three different body sites $(0.65 \%)$ in comparison with sampling of the anterior nares only $(0.96 \%)$. This could not be elaborated in more detail because we lack the MRSA culture method of the clinical laboratories other than the one of AZ Nikolaas.

Another option for reducing SSI after instrumented surgery is universal decolonization of patients. The latter approach may be more effective in reducing the rates of MRSA SSI infection in comparison to screening and selective decolonization and may also reduce hospitalization cost [20]. However, it should be kept in mind that widespread usage of decolonizing agents can induce an increased resistance against these agents [21] and may compromise the capacity of monitoring the emergence of new clones of $S$. aureus if previous screening is not performed [22]. Baratz et al. concluded that although an MRSA screening and decolonization protocol reduces the nasal carriage of MRSA in patients undergoing total joint arthroplasty, $22 \%$ of the patients remained colonized. This was partly explained by mupirocin resistance [23]. In our study, we did not observe regrowth of MRSA after decolonization procedures on patients. Denis et al. reported a MRSA sensitivity of $>90 \%$ for mupirocin in Belgium [24].

Of all searched risk factors, there was not a particular one which was remarkably present in MRSA colonized patients this may be due to the low MRSA prevalence. Noteworthy, eleven of the twelve patients had at least one risk factor. De Wouters et al. showed that selective screening of patients based on the presence of risk factors for MRSA colonization does not allow detecting the MRSA carriers when MRSA prevalence is low [25]. The identification of risk factors in individual patients may be interesting, but implementation of this time-consuming patient-based query is not standard.

There are different approaches for reducing the postoperative burden of MRSA SSI such as preoperative screening with subsequent decolonization, screening of high-risk patients with decolonization or universal decolonization. Hereby, the main goal should be a decrease of the MRSA colonization rate and prevention of postoperative complications caused by MRSA SSI. When comparing the abovementioned strategies, the local prevalence of MRSA carriage, a possible increase of resistance against decolonizing agents and the cost-effectiveness of the different approaches should be considered.

In conclusion, preoperative MRSA screening is a tool, which can be used to reduce possible complications caused by MRSA after surgery. In our study, the prevalence of MRSA carriage in elective, instrumented neurosurgery was $0.74 \%$ and no resistance was observed against our decolonization protocol. Considering the fact that an SSI requires long-term antibiotic treatment, which in case of MRSA 
Erik Van de Kelft (2019) Presurgical MRSA Screening and Subsequent Decolonization in Elective Instrumented Neurosurgery:

A Case Descriptive Study

infection should be administered intravenously, the cost of screening and decontamination seems justified.

\section{Competing interest}

The authors report no conflict of interest.

\section{Funding information}

This expenses for this study were covered by the department of neurosurgery

\section{Abbreviations}

MRSA: methicillin resistant staphylococcus aureus

MSSA: methicillin sensitive staphylococcus aureus

S. aureus: staphylococcus aureus

SSI: surgical side infection

\section{References}

1. Anderson, D.J., et al., (2009) Clinical and Financial Outcomes Due to Methicillin Resistant Staphylococcus aureus Surgical Site Infection: A Multi-Center Matched Outcomes Study. Plos One, 4(12). [Crossref]

2. Cassir, N., et al., (2015) Risk factors for surgical site infections after neurosurgery: A focus on the postoperative period. Am J Infect Control, 43(12): p. 1288-91. [Crossref]

3. Wertheim, H.F., et al., (2005) The role of nasal carriage in Staphylococcus aureus infections. Lancet Infect Dis, 5(12): p. 751-62. [Crossref]

4. Harbarth, S., et al., (2006) Evaluating the probability of previously unknown carriage of MRSA at hospital admission. Am J Med, 119(3): p. 275 e15-23. [Crossref]

5. Dorado-Garcia, A., et al., (2013) Risk factors for persistence of livestock-associated MRSA and environmental exposure in veal calf farmers and their family members: an observational longitudinal study. BMJ Open, 3(9): p. e003272. [Crossref]

6. Rogers, C., et al., (2014) Duration of colonization with methicillin-resistant Staphylococcus aureus in an acute care facility: a study to assess epidemiologic features. Am J Infect Control, 42(3): p. 249-53. [Crossref]

7. Couderc, C., et al., (2014) Fluoroquinolone use is a risk factor for methicillinresistant Staphylococcus aureus acquisition in long-term care facilities: a nested case-case-control study. Clin Infect Dis, 59(2): p. 206-15. [Crossref]

8. Zervou, F.N., et al., (2014) Prevalence of and risk factors for methicillin-resistant Staphylococcus aureus colonization in HIV infection: a meta-analysis. Clin Infect Dis, 59(9): p. 1302-11. [Crossref]

9. Nguyen, D.B., et al., (2013) Invasive methicillin-resistant Staphylococcus aureus infections among patients on chronic dialysis in the United States, 2005-2011. Clin Infect Dis, 57(10): p. 1393-400. [Crossref]

10. Centers for Disease, C. and Prevention, (2003) Methicillin-resistant staphylococcus aureus infections among competitive sports participants--Colorado, Indiana, Pennsylvania, and Los Angeles County, 2000-2003. MMWR Morb Mortal Wkly Rep, 52(33): p. 793-5. [Crossref]

11. Centers for Disease, C. and Prevention, (2001) Methicillin-resistant Staphylococcus aureus skin or soft tissue infections in a state prison--Mississippi, 2000. MMWR Morb Mortal Wkly Rep, 50(42): p. 919-22. [Crossref]

12. Centers for Disease, C. and Prevention, (2006) Methicillin-resistant Staphylococcus aureus skin infections among tattoo recipients--Ohio, Kentucky, and Vermont, 2004-2005. MMWR Morb Mortal Wkly Rep, 55(24): p. 677-9. [Crossref]

13. Diep, B.A., et al., (2008) Emergence of multidrug-resistant, community-associated, methicillin-resistant Staphylococcus aureus clone USA300 in men who have sex with men. Ann Intern Med, 148(4): p. 249-57. [Crossref]

14. Lefebvre, J., et al., (2017) Staphylococcus aureus screening and decolonization reduces the risk of surgical site infections in patients undergoing deep brain stimulation surgery. J Hosp Infect, 95(2): 144-147(1532-2939). [Crossref]

15. Slover, J., et al., (2011) Cost-effectiveness of a Staphylococcus aureus screening and decolonization program for high-risk orthopedic patients. J Arthroplasty, 26(3): p. 360-5. [Crossref]

16. den Heijer, C.D., et al., (2013) Prevalence and resistance of commensal Staphylococcus aureus, including meticillin-resistant $\mathrm{S}$ aureus, in nine European countries: a cross-sectional study. Lancet Infect Dis, 13(5): p. 409-15. [Crossref]
17. Kawabata, A., et al., (2017) Methicillin-resistant Staphylococcus Aureus Nasal Swab and Suction Drain Tip Cultures in 4573 Spinal Surgeries: Efficacy in Management of Surgical Site Infections. Spine (Phila Pa 1976), [Crossref]

18. Bode, L.G., et al., (2010) Preventing surgical-site infections in nasal carriers of Staphylococcus aureus. N Engl J Med, 362(1): p. 9-17. [Crossref]

19. Senn, L., et al., (2012) Which anatomical sites should be sampled for screening of methicillin-resistant Staphylococcus aureus carriage by culture or by rapid PCR test? Clinical Microbiology and Infection, 18(2): p. E31-E33. [Crossref]

20. Huang, S.S., et al., (2013) Targeted versus universal decolonization to prevent ICU infection. N Engl J Med, 368(24): p. 2255-65. [Crossref]

21. Hernandez-Porto, M., et al., (2014) Risk factors for development of methicillinresistant Staphylococcus aureus-positive clinical culture in nasal carriers after decolonization treatment. Am J Infect Control, 42(7): p. e75-9. [Crossref]

22. Humphreys, H., et al., (2016) Staphylococcus aureus and surgical site infections: benefits of screening and decolonization before surgery. $J$ Hosp Infect, 94(3): p. 295-304. [Crossref]

23. Baratz, M.D., et al., (2015) Twenty Percent of Patients May Remain Colonized With Methicillin-resistant Staphylococcus aureus Despite a Decolonization Protoco in Patients Undergoing Elective Total Joint Arthroplasty. Clin Orthop Relat Res, 473(7): p. 2283-90. [Crossref]

24. Denis, O., et al., (2006) In vitro activities of ceftobiprole, tigecycline, daptomycin, and 19 other antimicrobials against methicillin-resistant Staphylococcus aureus strains from a national survey of Belgian hospitals. Antimicrob Agents Chemother, 50(8): p. 2680-5. [Crossref]

25. de Wouters, S., et al., (2015) Selective Methicillin-Resistant Staphylococcus Aureus (MRSA) screening of a high risk population does not adequately detect MRSA carriers within a country with low MRSA prevalence. Acta Orthop Belg 81(4): p. 620-8. [Crossref]

\section{Citation:}

Vincent De La Porte, Veroniek Saegeman, Elise Willems, Charlotte Stolte and Erik Van de Kelft (2019) Presurgical MRSA Screening and Subsequent Decolonization in Elective Instrumented Neurosurgery: A Case Descriptive Study. J Clin Res Med Volume 2(5): 1-4. 